\title{
Improvements of the bounds for Ramanujan constant function
}

\author{
Hong-Hu Chu' ${ }^{1}$ Zhen-Hang Yang ${ }^{2}$, Wen Zhang ${ }^{3}$ and Yu-Ming Chu ${ }^{1 *}$
}

\section{"Correspondence:}

chuyuming2005@126.com

'School of Mathematics and

Computation Sciences, Hunan City

University, Yiyang, 413000, China

Full list of author information is

available at the end of the article

\begin{abstract}
In the article, we establish several inequalities for the Ramanujan constant function $R(x)=-2 \gamma-\psi(x)-\psi(1-x)$ on the interval $(0,1 / 2]$, where $\psi(x)$ is the classical psi function and $\gamma=0.577215 \ldots$ is the Euler-Mascheroni constant.
\end{abstract}

MSC: 33B15;26D07

Keywords: Ramanujan constant function; gamma function; psi function;

Euler-Mascheroni constant

\section{Introduction}

For $x>0$, the classical gamma function $\Gamma(x)$ and the psi function $\psi(x)$ are, respectively, defined by

$$
\Gamma(x)=\int_{0}^{\infty} t^{x-1} e^{-t} d t, \quad \psi(x)=\frac{\Gamma^{\prime}(x)}{\Gamma(x)}
$$

they satisfy

$$
\begin{aligned}
& \Gamma(x+1)=x \Gamma(x), \quad \psi(x+1)=\psi(x)+\frac{1}{x}, \\
& \psi(x)=\sum_{n=0}^{\infty} \frac{x-1}{(n+1)(n+x)}-\gamma, \quad \psi^{\prime}(x)=\sum_{n=0}^{\infty} \frac{1}{(n+x)^{2}}, \quad \psi^{\prime \prime}(x)=-2 \sum_{n=0}^{\infty} \frac{1}{(n+x)^{3}}, \\
& \psi(1)=-\gamma, \quad \psi\left(\frac{1}{2}\right)=-2 \log 2-\gamma,
\end{aligned}
$$

where $\gamma=\lim _{n \rightarrow \infty}\left(\sum_{k=1}^{n} 1 / k-\log n\right)=0.577215 \cdots$ is the Euler-Mascheroni constant.

It is well known that the gamma and psi functions have many applications in the areas of mathematics, physics, and engineering technology. Recently, the bounds for the gamma and psi functions have attracted the interest of many researchers. In particular, many remarkable inequalities for the psi function $\psi(x)$ can be found in the literature [1-15].

Let $x \in(0,1 / 2]$. Then the Ramanujan constant function $R(x)[16]$ is given by

$$
R(x)=-2 \gamma-\psi(x)-\psi(1-x)
$$

(c) 2016 Chu et al. This article is distributed under the terms of the Creative Commons Attribution 4.0 International License (http://creativecommons.org/licenses/by/4.0/), which permits unrestricted use, distribution, and reproduction in any medium, provided you give appropriate credit to the original author(s) and the source, provide a link to the Creative Commons license, and indicate if changes were made. 
Very recently, Wang et al. [17] proved that the double inequality

$$
\frac{R^{2}(x)}{\left(1+x-x^{2}\right) R(x)-1}<\frac{\pi}{\sin (\pi x)}<\left(1+x-x^{2}\right) R(x)
$$

holds for all $x \in(0,1 / 2]$.

The main purpose of this paper is to improve inequality (1.2).

\section{Lemmas}

In order to prove our main results we need several lemmas, which we present in this section.

Lemma 2.1 (See $[18,19])$ Let $-\infty<a<b<\infty, f, g:[a, b] \rightarrow \mathbb{R}$ be continuous on $[a, b]$ and differentiable on $(a, b)$, and $g^{\prime}(x) \neq 0$ on $(a, b)$. Then the functions

$$
\frac{f(x)-f(a)}{g(x)-g(a)}
$$

and

$$
\frac{f(x)-f(b)}{g(x)-g(b)}
$$

both are increasing (decreasing) on $(a, b)$ if $f^{\prime}(x) / g^{\prime}(x)$ is increasing (decreasing) on $(a, b)$. If $f^{\prime}(x) / g^{\prime}(x)$ is strictly monotone, then the monotonicity in the conclusion is also strict.

Lemma 2.2 (See [20]) The double inequality

$$
\frac{x^{2}+1}{x+1}<\Gamma(x+1)<\frac{x^{2}+2}{x+2}
$$

holds for all $x \in(0,1)$.

Lemma 2.3 (See [21], Section 3, in the proof of Theorem 5, pp. 2500-2502) Let $x \in$ $(0, \pi / 2), k, n \in \mathbb{N}$, the sequence $\left\{a_{k}\right\}_{k=0}^{\infty}$ and function $F_{n}(x)$ be, respectively, defined by

$$
\begin{aligned}
& a_{0}=\frac{2}{\pi}, \quad a_{1}=\frac{1}{\pi^{3}}, \\
& a_{k+1}=\frac{2 k+1}{2(k+1) \pi^{2}} a_{k}-\frac{1}{16 k(k+1) \pi^{2}} a_{k-1} \quad(k \geq 1), \\
& F_{n}(x)=\frac{\frac{\sin x}{x}-\sum_{k=0}^{n} a_{k}\left(\pi^{2}-4 x^{2}\right)^{k}}{\left(\pi^{2}-4 x^{2}\right)^{n+1}} .
\end{aligned}
$$

Then $F_{n}(x)$ is strictly decreasing from $(0, \pi / 2)$ onto $\left(a_{n+1},\left(1-\sum_{k=0}^{n} a_{k} \pi^{2 k}\right) / \pi^{2 n+2}\right)$.

Lemma 2.4 Let $k, n \in \mathbb{N},\left\{a_{k}\right\}_{k=0}^{\infty}$ be defined by (2.1) and (2.2), and $\left\{b_{k}\right\}_{k=0}^{\infty}$ be defined by

$$
\begin{aligned}
& b_{0}=0, \quad b_{1}=\frac{1}{4 \pi}, \\
& b_{k+1}=\frac{2 k-1}{2(k+1) \pi^{2}} b_{k}-\frac{1}{16 k(k+1) \pi^{2}} b_{k-1} \quad(k \geq 1) .
\end{aligned}
$$

Then $a_{k}=8(k+1) b_{k+1}$ for all $k \in \mathbb{N}$. 
Proof We use mathematical induction to prove Lemma 2.4. From (2.1), (2.4), and (2.5) we clearly see that Lemma 2.4 holds for $k=0$ and $k=1$.

Suppose that $k_{0} \geq 1$ and

$$
a_{k}=8(k+1) b_{k+1}
$$

holds for all $k \leq k_{0}$. Then it follows from (2.2), (2.5), and (2.6) that

$$
\begin{aligned}
a_{k_{0}+1} & =\frac{2 k_{0}+1}{2\left(k_{0}+1\right) \pi^{2}} a_{k_{0}}-\frac{1}{16 k_{0}\left(k_{0}+1\right) \pi^{2}} a_{k_{0}-1} \\
& =\frac{2 k_{0}+1}{2\left(k_{0}+1\right) \pi^{2}} \times 8\left(k_{0}+1\right) b_{k_{0}+1}-\frac{1}{16 k_{0}\left(k_{0}+1\right) \pi^{2}} \times 8 k_{0} b_{k_{0}} \\
& =8\left(k_{0}+2\right) b_{k_{0}+2}=8\left[\left(k_{0}+1\right)+1\right] b_{\left(k_{0}+1\right)+1} .
\end{aligned}
$$

Equation (2.7) shows that (2.6) also holds for $k=k_{0}+1$. Therefore, Lemma 2.4 follows from (2.7) and the induction hypothesis (2.6).

Lemma 2.5 The double inequality

$$
\begin{aligned}
& \frac{1}{4 \pi}\left(\pi^{2}-4 x^{2}\right)+\frac{1}{16 \pi^{3}}\left(\pi^{2}-4 x^{2}\right)^{2}+\frac{12-\pi^{2}}{384 \pi^{5}}\left(\pi^{2}-4 x^{2}\right)^{3} \\
& \quad<\cos x<\frac{1}{4 \pi}\left(\pi^{2}-4 x^{2}\right)+\frac{1}{16 \pi^{3}}\left(\pi^{2}-4 x^{2}\right)^{2}+\frac{16-5 \pi}{16 \pi^{6}}\left(\pi^{2}-4 x^{2}\right)^{3}
\end{aligned}
$$

holds for all $x \in(0, \pi / 2)$.

Proof Let $x \in(0, \pi / 2), k, n \in \mathbb{N},\left\{a_{k}\right\}_{k=0}^{\infty}$ and $\left\{b_{k}\right\}_{k=0}^{\infty}$ be, respectively, defined by (2.1), (2.2), (2.4), and (2.5), $F_{n}(x)$ be defined by (2.3), and $f_{n}(x)$ and $g_{n}(x)$ be defined by

$$
f_{n}(x)=\cos x-\sum_{k=0}^{n} b_{k}\left(\pi^{2}-4 x^{2}\right)^{k}, \quad g_{n}(x)=\left(\pi^{2}-4 x^{2}\right)^{n+1} .
$$

Then it follows from (2.1)-(2.5), Lemma 2.4, and equation (2.9) that

$$
\begin{aligned}
& a_{2}=\frac{12-\pi^{2}}{16 \pi^{5}}, \quad b_{2}=\frac{1}{16 \pi^{3}}, \quad \frac{f_{2}\left(0^{+}\right)}{g_{2}\left(0^{+}\right)}=\frac{1-\sum_{k=0}^{2} b_{k} \pi^{2 k}}{\pi^{6}}=\frac{16-5 \pi}{16 \pi^{6}}, \\
& \frac{f_{n}\left(0^{+}\right)}{g_{n}\left(0^{+}\right)}=\frac{1-\sum_{k=0}^{n} b_{k} \pi^{2 k}}{\pi^{2 n+2}}, \\
& \frac{f_{n}(x)}{g_{n}(x)}=\frac{f_{n}(x)-f_{n}\left(\frac{\pi}{2}\right)}{g_{n}(x)-g_{n}\left(\frac{\pi}{2}\right)}, \\
& \frac{f_{n}^{\prime}(x)}{g_{n}^{\prime}(x)}=\frac{\frac{\sin x}{x}-\sum_{k=0}^{n-1} 8(k+1) b_{k+1}\left(\pi^{2}-4 x^{2}\right)^{k}}{8(n+1)\left(\pi^{2}-4 x^{2}\right)^{n}}=\frac{F_{n-1}(x)}{8(n+1)} .
\end{aligned}
$$

From Lemma 2.1, Lemma 2.3, (2.12), and (2.13) we clearly see that

$$
\frac{a_{n}}{8(n+1)}=\lim _{x \rightarrow \pi / 2^{-}} \frac{f_{n}(x)}{g_{n}(x)}<\frac{f_{n}(x)}{g_{n}(x)}<\lim _{x \rightarrow 0^{+}} \frac{f_{n}(x)}{g_{n}(x)}
$$

for all $x \in(0, \pi / 2)$. 
Equations (2.9) and (2.11) together with inequality (2.14) lead to the conclusion that

$$
\frac{a_{n}}{8(n+1)}<\frac{\cos x-\sum_{k=0}^{n} b_{k}\left(\pi^{2}-4 x^{2}\right)^{k}}{\left(\pi^{2}-4 x^{2}\right)^{n+1}}<\frac{1-\sum_{k=0}^{n} b_{k} \pi^{2 k}}{\pi^{2 n+2}}
$$

for all $x \in(0, \pi / 2)$.

Letting $n=2$, then inequality (2.8) follows easily from (2.4), (2.10), and (2.15).

Remark 2.1 We clearly see that both the first and the second inequalities in (2.8) become to equations if $x=\pi / 2$. If $x=0$, then the first inequality of (2.8) also holds and the second inequality of (2.8) becomes to equation.

Lemma 2.6 Let $n \in \mathbb{N}$ and $R(x)$ be the Ramanujan constant function given by (1.1). Then the double inequality

$$
\begin{aligned}
& \sum_{k=0}^{n-1} \frac{2 k+1}{(k+x)(k+1-x)}+2 \psi(1)-2 \psi\left(n+\frac{1}{2}\right) \\
& \quad \leq R(x)<\sum_{k=0}^{n-1} \frac{2 k+1}{(k+x)(k+1-x)}+2 \psi(1)-\psi(n)-\psi(n+1)
\end{aligned}
$$

holds for all $x \in(0,1 / 2]$ and $n \geq 1$.

Proof Let $n \in \mathbb{N}, x \in(0,1 / 2]$, and

$$
h_{n}(x)=2 \psi(1)-\psi(n+x)-\psi(n+1-x)
$$

Then (1.1) and (2.17) together with the mean value theorem lead to

$$
\begin{aligned}
& h_{n}(x)=R(x)-\sum_{k=0}^{n-1} \frac{1}{k+x}-\sum_{k=0}^{n-1} \frac{1}{k+1-x}=R(x)-\sum_{k=0}^{n-1} \frac{2 k+1}{(k+x)(k+1-x)}, \\
& h_{n}^{\prime}(x)=-\psi^{\prime}(n+x)+\psi^{\prime}(n+1-x)=(1-2 x) \psi^{\prime \prime}[n+x+\theta(1-2 x)]<0
\end{aligned}
$$

for $x \in(0,1 / 2]$, where $\theta \in(0,1)$.

It follows from (2.17) and (2.19) that

$$
2 \psi(1)-2 \psi\left(n+\frac{1}{2}\right)=h_{n}\left(\frac{1}{2}\right) \leq h_{n}(x)<h_{n}\left(0^{+}\right)=2 \psi(1)-\psi(n)-\psi(n+1) .
$$

Therefore, Lemma 2.6 follows from (2.18) and (2.20).

Lemma 2.7 Let $A(t)$ and $B(t)$ be defined by

$$
A(t)=\frac{8\left(320 t^{3}-1,936 t^{2}+1,292 t+945\right)^{2}}{3(1-4 t)(9-4 t)(25-4 t)\left(-1,280 t^{4}+9,728 t^{3}-18,208 t^{2}+8,896 t+3,375\right)}
$$


and

$$
B(t)=\frac{236}{3(125-28 t)}+\frac{4}{1-4 t}-\frac{12}{9-4 t}-\frac{4}{25-4 t} .
$$

Then $0<A(t)<B(t)$ for $t \in[0,1 / 4)$.

Proof We clearly see that

$$
A(t)>0, \quad 880 t^{2}-6,488 t+10,207>0
$$

and

$$
-1,280 t^{4}+9,728 t^{3}-18,208 t^{2}+8,896 t+3,375>0
$$

for $t \in[0,1 / 4)$.

Therefore, Lemma 2.7 follows from (2.23) and (2.24) together with the elaborated computations result

$$
A(t)-B(t)=-\frac{32 t(1-4 t)\left(880 t^{2}-6,488 t+10,207\right)}{(125-28 t)\left(-1,280 t^{4}+9,728 t^{3}-18,208 t^{2}+8,896 t+3,375\right)} .
$$

Lemma 2.8 Let $B(t)$ be defined by (2.22). Then

$$
B(t)<\frac{\pi}{\cos (\sqrt{t} \pi)} .
$$

for $t \in[0,1 / 4)$.

Proof From (2.22) we clearly see that $B(0)=1,176 / 375=3.136<\pi$, which implies that inequality (2.26) holds for $t=0$.

Let $t \in(0,1 / 4)$. Then $\sqrt{t} \pi \in(0, \pi / 2)$ and the second inequality in (2.8) leads to

$$
\cos (\sqrt{t} \pi)<\frac{\pi}{4}(1-4 t)+\frac{\pi}{16}(1-4 t)^{2}+\frac{16-5 \pi}{16}(1-4 t)^{3} .
$$

It follows from (2.22) and (2.27) that

$$
\begin{aligned}
B(t) & -\frac{\pi}{\cos (\sqrt{t} \pi)} \\
< & \frac{236}{3(125-28 t)}+\frac{4}{1-4 t}-\frac{12}{9-4 t}-\frac{4}{25-4 t} \\
& -\frac{\pi}{\frac{\pi}{4}(1-4 t)+\frac{\pi}{16}(1-4 t)^{2}+\frac{16-5 \pi}{16}(1-4 t)^{3}} \\
= & \left(4 ( 1 - 4 t ) \left[(1,024-320 \pi) t^{3}+(3,864 \pi-12,416) t^{2}+(32,128-9,750 \pi) t\right.\right. \\
& +264,600-84,375 \pi]) \\
& /\left(3(9-4 t)(25-4 t)(125-28 t)\left[(64-20 \pi) t^{2}+(9 \pi-32) t+4\right]\right) .
\end{aligned}
$$


Note that

$$
\begin{aligned}
& \frac{(1-4 t)}{(9-4 t)(25-4 t)(125-28 t)}>0, \\
& (64-20 \pi) t^{2}+(9 \pi-32) t+4>\frac{9 \pi-32}{4}+4=\frac{9 \pi-16}{4}>0, \\
& (1,024-320 \pi) t^{3}+(3,864 \pi-12,416) t^{2}+(32,128-9,750 \pi) t+264,600-84,375 \pi \\
& \quad<\frac{1,024-320 \pi}{64}+\frac{32,128-9,750 \pi}{4}+264,600-84,375 \pi \\
& \quad=272,648-86,817.5 \times \pi=-97.2202 \ldots
\end{aligned}
$$

for $t \in(0,1 / 4)$.

Therefore, Lemma 2.8 follows from (2.28)-(2.31).

\section{Main results}

Theorem 3.1 Let $R(x)$ be the Ramanujan constant function given by $(1.1)$ and $C(x)$ be defined by

$$
C(x)=\frac{2\left[320\left(\frac{1}{2}-x\right)^{6}-1,936\left(\frac{1}{2}-x\right)^{4}+1,292\left(\frac{1}{2}-x\right)^{2}+945\right]}{3\left[1-4\left(\frac{1}{2}-x\right)^{2}\right]\left[9-4\left(\frac{1}{2}-x\right)^{2}\right]\left[25-4\left(\frac{1}{2}-x\right)^{2}\right]} .
$$

Then

$$
4 \log 2 \leq \frac{1}{x(1-x)}-4+4 \log 2 \leq R(x)<C(x)
$$

for $x \in(0,1 / 2]$.

Proof Let $n=1$, then the first inequality of (2.16) leads

$$
\begin{aligned}
R(x) & \geq \frac{1}{x(1-x)}+2\left[\psi(1)-\psi\left(\frac{3}{2}\right)\right]=\frac{1}{x(1-x)}+2\left[\psi(1)-\psi\left(\frac{1}{2}\right)-2\right] \\
& =\frac{1}{x(1-x)}-4+4 \log 2 \geq 4 \log 2
\end{aligned}
$$

for $x \in(0,1 / 2]$.

Let $n=3$ and $x \in(0,1 / 2)$, then (3.1) and the second inequality of (2.16) give

$$
\begin{aligned}
R(x) & <\sum_{k=0}^{2} \frac{2 k+1}{(k+x)(k+1-x)}+2 \psi(1)-\psi(3)-\psi(4) \\
& =\sum_{k=0}^{2} \frac{2 k+1}{\left(k+\frac{1}{2}\right)^{2}-\left(\frac{1}{2}-x\right)^{2}}+2 \psi(1)-2\left[\psi(1)+\frac{3}{2}\right]-\frac{1}{3} \\
& =\frac{1}{\frac{1}{4}-\left(\frac{1}{2}-x\right)^{2}}+\frac{3}{\frac{9}{4}-\left(\frac{1}{2}-x\right)^{2}}+\frac{5}{\frac{25}{4}-\left(\frac{1}{2}-x\right)^{2}}-\frac{10}{3} \\
& =\frac{2\left[320\left(\frac{1}{2}-x\right)^{6}-1,936\left(\frac{1}{2}-x\right)^{4}+1,292\left(\frac{1}{2}-x\right)^{2}+945\right]}{3\left[1-4\left(\frac{1}{2}-x\right)^{2}\right]\left[9-4\left(\frac{1}{2}-x\right)^{2}\right]\left[25-4\left(\frac{1}{2}-x\right)^{2}\right]}=C(x) .
\end{aligned}
$$


If $x=1 / 2$, then (1.1) and (3.1) lead to

$$
R\left(\frac{1}{2}\right)=4 \log 2=2.7725 \cdots<C\left(\frac{1}{2}\right)=\frac{378}{135}=2.8
$$

Theorem 3.2 Let $R(x)$ be the Ramanujan constant function, given by (1.1), and the function $A(t)$ be defined by (2.21). Then the inequality

$$
\frac{R^{2}(x)}{\left(1+x-x^{2}\right) R(x)-1}<A\left[\left(\frac{1}{2}-x\right)^{2}\right]
$$

for all $x \in(0,1 / 2]$.

Proof Let $C(x)$ be defined by (3.1). Then from (3.2) and $4 \log 2=2.7725 \cdots>2$ together with

$$
\frac{\partial\left[\frac{y}{\left(1+x-x^{2}\right) y-1}\right]}{\partial y}=\frac{[x(1-x) y+(y-2)] y}{\left[\left(1+x-x^{2}\right) y-1\right]^{2}}>0
$$

for $y>2$ and $x \in(0,1 / 2]$ we clearly see that

$$
\frac{R^{2}(x)}{\left(1+x-x^{2}\right) R(x)-1}<\frac{C^{2}(x)}{\left(1+x-x^{2}\right) C(x)-1} .
$$

for $x \in(0,1 / 2]$.

Elaborated computations give

$$
\frac{C^{2}(x)}{\left(1+x-x^{2}\right) C(x)-1}=A\left[\left(\frac{1}{2}-x\right)^{2}\right]
$$

Therefore, Theorem 3.2 follows from (3.4) and (3.5).

Theorem 3.3 Let $R(x)$ be the Ramanujan constant function given by (1.1). Then

$$
R(x)>\frac{\pi}{\left(1+x-x^{2}\right) \sin (\pi x)}+\frac{125 \log 2-81}{4\left(1+x-x^{2}\right)\left(6+x-x^{2}\right)}
$$

for $x \in(0,1 / 2]$.

Proof It is well known that

$$
\Gamma(x) \Gamma(1-x)=\frac{\pi}{\sin (\pi x)}
$$

for $x \in(0,1)$.

It follows from Lemma 2.2 and (3.2) that

$$
\begin{aligned}
& \frac{\pi}{\sin (\pi x)}-\left(1+x-x^{2}\right) R(x) \\
& \quad=\Gamma(x) \Gamma(1-x)-\left(1+x-x^{2}\right) R(x)=\frac{\Gamma(x+1)}{x} \frac{\Gamma[(1-x)+1]}{1-x}-\left(1+x-x^{2}\right) R(x)
\end{aligned}
$$




$$
\begin{aligned}
& <\frac{x^{2}+2}{x(x+2)} \frac{(1-x)^{2}+2}{(1-x)(3-x)}-\left(1+x-x^{2}\right)\left[\frac{1}{x(1-x)}+4 \log 2-4\right] \\
& =\frac{4(1-\log 2) x(1-x)\left(7+x-x^{2}\right)-(24 \log 2-13)}{6+x-x^{2}}
\end{aligned}
$$

for $x \in(0,1 / 2]$.

Note that

$$
x(1-x)\left(7+x-x^{2}\right) \leq \frac{1}{4} \times \frac{29}{4}=\frac{29}{16}
$$

for $x \in(0,1 / 2]$.

It follows from (3.8) and (3.9) that

$$
\frac{\pi}{\sin (\pi x)}-\left(1+x-x^{2}\right) R(x)<-\frac{125 \log 2-81}{4\left(6+x-x^{2}\right)}
$$

for $x \in(0,1 / 2]$.

Therefore, Theorem 3.3 follows easily from (3.10).

Corollary 3.1 Let $R(x)$ be the Ramanujan constant function given by (1.1). Then

$$
R(x)>\frac{\pi}{\left(1+x-x^{2}\right) \sin (\pi x)}+4 \log 2-\frac{324}{125}=\frac{\pi}{\left(1+x-x^{2}\right) \sin (\pi x)}+0.18058872 \ldots
$$

for $x \in(0,1 / 2]$.

Proof Corollary 3.1 follows easily from (3.6) and

$$
4\left(1+x-x^{2}\right)\left(6+x-x^{2}\right) \leq 4 \times\left(1+\frac{1}{4}\right)\left(6+\frac{1}{4}\right)=\frac{125}{4}
$$

for $x \in(0,1 / 2]$.

Remark 3.1 Let $A(t)$ and $B(t)$ be, respectively, defined by (2.21) and (2.22). Then it follows from Lemmas 2.7 and 2.8 that

$$
A\left[\left(\frac{1}{2}-x\right)^{2}\right]<B\left[\left(\frac{1}{2}-x\right)^{2}\right]<\frac{\pi}{\cos \left[\pi\left(\frac{1}{2}-x\right)\right]}=\frac{\pi}{\sin (\pi x)}
$$

for $x \in(0,1 / 2]$.

Therefore, inequality (3.3) is an improvement of the first inequality given by (1.2).

Remark 3.2 We clearly see that inequality (3.6) is an improvement of the second inequality given by (1.2).

The authors declare that they have no competing interests. 


\section{Author details}

'School of Mathematics and Computation Sciences, Hunan City University, Yiyang, 413000, China. ${ }^{2}$ Customer Service Center, State Grid Zhejiang Electric Power Research Institute, Hangzhou, 310009, China. ${ }^{3}$ Albert Einstein College of Medicine, Yeshiva University, New York, NY 10033, United States.

\section{Acknowledgements}

The research was supported by the Natural Science Foundation of China under Grants 11371125, 61374086 and 11401191, and the Natural Science Foundation of Zhejiang Province under Grant LY13A010004.

Received: 19 April 2016 Accepted: 2 August 2016 Published online: 12 August 2016

\section{References}

1. Alzer, H: On some inequalities for the gamma and psi functions. Math. Comput. 66(217), 373-389 (1997)

2. Palumbo, B: Determinantal inequalities for the psi function. Math. Inequal. Appl. 2(2), 223-231 (1999)

3. Qiu, S-L, Vuorinen, M: Some properties of the gamma and psi functions, with applications. Math. Comput. 74(250), 723-742 (2005)

4. Simić, S: Inequalities for $\Psi$ function. Math. Inequal. Appl. 10(1), 45-48 (2007)

5. Wu, L-L, Chu, Y-M: An inequality for the psi functions. Appl. Math. Sci. 2(9-12), 545-550 (2008)

6. Chu, Y-M, Zhang, X-M, Tang, X-M: An elementary inequality for psi function. Bull. Inst. Math. Acad. Sin. 3(3), 373-380 (2008)

7. $\mathrm{Wu}, \mathrm{L}-\mathrm{L}, \mathrm{Chu}, \mathrm{Y}-\mathrm{M}$, Tang, X-M: Inequalities for the generalized logarithmic mean and psi functions. Int. J. Pure Appl. Math. 48(1), 117-122 (2008)

8. Chen, C-P, Srivastava, HM: Some inequalities and monotonicity properties associated with the gamma and psi functions and the Barnes G-function. Integral Transforms Spec. Funct. 22(1), 1-15 (2011)

9. Mortici, C: Accurate estimates of the gamma function involving the PSI function. Numer. Funct. Anal. Optim. 32(4), 469-476 (2011)

10. Batir, N: Sharp bounds for the psi function and harmonic numbers. Math. Inequal. Appl. 14(4), 917-925 (2011)

11. Chen, C-P, Batir, N: Some inequalities and monotonicity properties associated with the gamma and psi function. Appl. Math. Comput. 218(17), 8217-8225 (2012)

12. Guo, B-N, Qi, F: Sharp inequalities for the psi function and harmonic numbers. Analysis 34(2), 201-208 (2014)

13. Elezović, N: Estimations of psi function and harmonic numbers. Appl. Math. Comput. 258, 192-205 (2015)

14. Yang, Z-H, Chu, Y-M, Zhang, X-H: Sharp bounds for psi function. Appl. Math. Comput. 268(17), 1055-1063 (2015)

15. Chen, C-P: Inequalities and asymptotic expansions for the psi function and the Euler-Mascheroni constant. J. Number Theory 163, 596-607 (2016)

16. Qiu, S-L, Vuorinen, M: Special functions in geometric function theory. In: Handbook of Complex Analysis: Geometric Function Theory, vol. 2, pp. 621-659. Elsevier, Amsterdam (2005)

17. Wang, M-K, Chu, Y-M, Qiu, S-L: Sharp bounds for generalized elliptic integrals of the first kind. J. Math. Anal. Appl. 429(2), 744-757 (2015)

18. Anderson, GD, Vamanamurthy, MK, Vuorinen, M: Conformal Invariants, Inequalities, and Quasiconformal Maps. Wiley, New York (1997)

19. Anderson, GD, Qiu, S-L, Vamanamurthy, MK, Vuorinen, M: Generalized elliptic integrals and modular equations. Pac. J. Math. 192(1), 1-37 (2000)

20. Ivády, P: A note on a gamma function inequality. J. Math. Inequal. 3(3), 227-236 (2009)

21. Zhu, L: A general refinement of Jordan-type inequality. Comput. Math. Appl. 55(11), 2498-2505 (2008)

\section{Submit your manuscript to a SpringerOpen ${ }^{\circ}$ journal and benefit from:}

- Convenient online submission

Rigorous peer review

- Immediate publication on acceptance

- Open access: articles freely available online

- High visibility within the field

- Retaining the copyright to your article 